\title{
Design and Calculation of the Transmission System for Sintering Furnace
}

\author{
Shaohua Tong ${ }^{\mathrm{a}}$, Guiqin $\mathrm{Li}^{\mathrm{b}}$, Lixin $\mathrm{Lu}^{\mathrm{c}}$ and Shuai Guo ${ }^{\mathrm{d}}$ \\ Shanghai Key Laboratory of Mechanical Automation and Robotics, Shanghai University, Shanghai, \\ 200072, China \\ axiaotong12@shu.edu.cn, 'leechingcn@hotmail.com, 'lulixin@shu.edu.cn, 'guoshuai@shu.edu.cn
}

Keywords: Sintering furnace; Transmission system; High-speed and running stability

\begin{abstract}
The design and calculation method about the belt transmission system of sintering furnace for solar cell is put forward to obtain rapid transmission speed and running stability of the belt. The structure of the power source is determined and the deceleration device is designed based on the calculation of the driving force. Focus on designing of mesh belt tensioning device and deviation-rectifying device to ensure the running stability of the transmission system. The sintering furnace designed has already manufactured and operates well, and its transmission system achieves the design request as well.
\end{abstract}

\section{Introduction}

The process of sintering is a major step for fabrication of solar cell, it makes the crystalline silicon substrate has photoelectric conversion function by controlling the sintering process curve which is mainly influenced by heating temperature and speed of transmission belt [1,2]. The mesh belt transmission system is an important part of sintering furnace, and the speed of mesh belt transmission system directly influences the power distribution of each section and the chamber structure in the sintering furnace. The mesh belt conveyor speed required of infrared heating sintering furnace for solar cells is $600 \mathrm{~mm} / \mathrm{min}$, while common mesh belt furnace usually operates at the speed of only 200 $\mathrm{mm} / \mathrm{min}[3,4]$. The high-speed sintering will make the running stability of the transmission belt worse, and furthermore affect the quality and the yield of sintering products.

To solve the problem mentioned above, the mesh belt transmission system of the sintering furnace is designed. By design and calculation of the power source of the mesh belt transmission system and design of the mesh belt tensioning device and deviation-rectifying device, the high-speed and running stability of the transmission system for sintering furnace is realized.

\section{Design of drive system for sintering furnace}

Sintering furnace selects metal transmission mesh belt, it should be fulfilled the following requirements [3]:

a) Be applicable to longer distance transmission; be flexibility, buffer, shock absorption, stability and low noise and no play.

b) The transmission system has been set up tension device in which the mesh belt wheel shall be able to withstand the greater axis force.

c) The deformation can't be too big when the belt go through the high heating sintering area, and the mesh belt has the properties of resistant to sudden change in operating temperature.

According to the requirements above, the overall structure of the transmission system in sintering furnace is designed as shown in Fig. 1. The transmission system of sintering furnace is composed by main transmission mechanism, follower mechanism, transition mechanism and mesh belt, four major parts. The driving member that is set in the workbench at the end of the sintering furnace is the power source of whole system and includes driving mechanism, decelerate components and tensioning device. The follower mechanism is set in the workbench at inlet and outlet of sintering furnace, including the components such as driven roller, mesh belt deviation-rectifying device, guiding mesh 
belt running perform in a required manner. The mesh belt with balanced knitting structure provides both advantages of deformation controlled and elongation decreased as it runs.

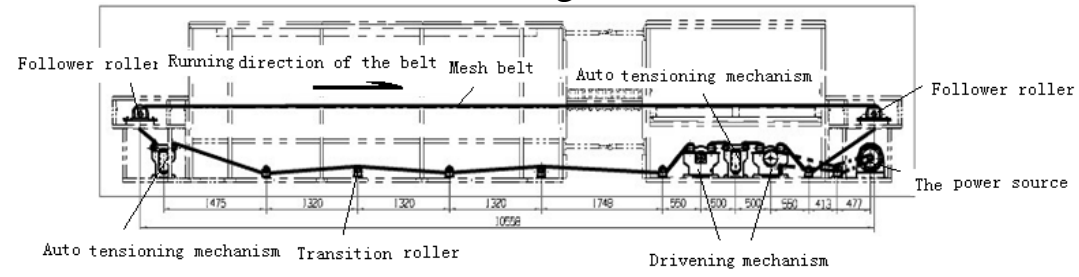

Fig. 1 Structure of sintering furnace transmission system

\section{Design and calculation of the power for mesh belt transmission system}

Calculation of Motor Power. When the high temperature metal mesh belt is running, the circumference driving force $\left(F_{U}\right)$ on transmission roller is the sum of all resistances in the operation process.

For the transmission system of sintering furnace for solar cell, the circumferential driving force is described as

$F_{U}=C f L g\left(q_{R 1}+q_{R 2}+q_{R 3}+2 q_{B}+q_{G} \cos \delta\right)+F_{S 1}+F_{S 2}$.

Where $f$ is simulation friction coefficient which is selected according to the working conditions and the level of manufacturing.

$L$ is the length of the conveying system (the center distance of head and tail roller), $1.558 \mathrm{~m}$;

$g$ denotes the gravity acceleration, $g=9.8 \mathrm{~m} / \mathrm{s}^{2}$;

$q_{R 1}$ denotes quality of rotating part per meter in transition wheel, $\mathrm{kg} / \mathrm{m}$;

$q_{R 2}$ denotes the quality of rotating part per meter in bend wheel, $\mathrm{kg} / \mathrm{m}$;

$q_{R 3}$ denotes the quality of rotating part per meter in two rolling wheels fore and aft, $\mathrm{kg} / \mathrm{m}$;

$q_{B}$ denotes the quality of transmission belt per meter, $\mathrm{kg} / \mathrm{m}$;

$q_{G}$ denotes the quality of material carried on mesh belt per meter $\mathrm{kg} / \mathrm{m}$;

$\delta$ denotes the inclination of the belt transmission system, and for horizontal transmission system, $\cos \delta=1$;

$F_{S 1}$ denotes friction resistance caused by deviation-rectifying device, $N$;

$F_{S 2}$ denotes the resistance caused by sweeper, unloader and turn over branch of conveyor, $N$;

$\mathrm{C}$ is introduced to denote the effect of the additional resistance on the belt transmission system.

When the length of mesh belt transmission system is less than $40 \mathrm{~m}$, the coefficient $\mathrm{C}$ can be 2.4 , the simulation friction coefficient $f$ is 0.02 ; the quality of rotating part of transition wheel is $28 \mathrm{~kg}$; the quality of rotating part of bending wheel is $72 \mathrm{~kg}$; and the quality of rotating part of fore and aft rolling wheels is $145 \mathrm{~kg}$. Thus

$$
\begin{aligned}
& q_{R 1}=(28 \times 5) / 1.32=106.06 \mathrm{~kg} / \mathrm{m} . \\
& q_{R 2}=(72 \times 6) / 0.25=1728 \mathrm{~kg} / \mathrm{m} . \\
& q_{R 3}=(145 \times 2) / 0.4=725 \mathrm{~kg} / \mathrm{m} .
\end{aligned}
$$

The quality per meter of the mesh belt $q_{B}=3.2 \mathrm{~kg} / \mathrm{m}$, which is given by the mesh belt producer; the quality of transmission belt per meter $q_{G}=2.73 \mathrm{~kg} / \mathrm{m}$; the rolling friction between deviation-rectifying device and mesh belt is negligible, $F_{S 1}=0$.

According to the contact area between the sweeper and mesh belt, based on calculation method of special resistance, thus the following value is obtained

$F_{S 2}=A_{p u}=2 \times 0.01 \times 1 \times 10^{6} \times 0.6=720 N$.

Put the values mentioned above into Eq. 1, 
$F_{U}=2.4 \times 0.02 \times 10.558 \times 9.8 \times(106.06+1728+725+2 \times 3.2+2.73)+0+720=13474.57 \mathrm{~N}$.

The driving power of the mesh belt transmission system is described as

$P_{A}=F_{U} V / 1000(k W)$.

In which the mesh belt speed $V=0.075 \mathrm{~m} / \mathrm{s}$. Put it into Eq. 2, we get the drive power

$P_{A}=1.02(k W)$.

Because output rotational speed of motor should pass through the clutch, reducer and a series of components to driving roller, so the transfer power of mesh belt transmission system effect by the transfer efficiency. Therefore, the practical power of the motor is

$P_{M}=P_{A} / \eta$.

Where $\eta=\eta_{1} \eta_{2} \eta_{3}$ (clutch efficiency $\eta_{1}=0.97$, reducer efficiency $\eta_{2}=0.94$, belt transmission efficiency $\left.\eta_{3}=0.9\right)$. So

$$
P_{M}=1.243(k W) \text {. }
$$

According to the motor power obtained above, the frequency conversion motor with cycloid pinwheel reducer is selected in the mesh belt transmission system and its power is $1.5 \mathrm{KW}$. The motor has the merits of simple structure, smooth operation, simple speed regulation, etc.

Design of Deceleration Mechanism. In order to decelerate the speed of mesh belt, a series of deceleration mechanisms are used. As shown in Fig. 2, the system uses motor 1 as a power source, the output rotational speed of motor is decelerated by the cycloid pinwheel reducer in motor and then passing through the first v-belt driving device 2 to the worm gear reducer 3 , then forces the driving roller 6 rotate by v-belt driving device 5 . The two driving rollers, with a coating of plastic to increase friction, transmit motion by synchronous belt. Through above process, the conveyer function on mesh belt is implemented. To ensure the transmission system running stability, gravity tensioning roller 8 is set between the two of driving rollers. The torque is delivered from reducer 3 to $\mathrm{V}$-belt transmission 5 by electromagnetic clutch 4, which is also as an overload protection. The rotational speed of motor can be changed by varying frequency of power by adjusting frequency converser, so the speed of belt can be obtained in the continuously adjustable range.

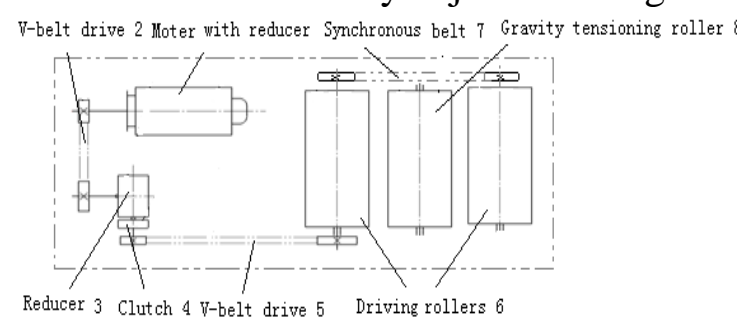

Fig. 2 Diagram of deceleration device

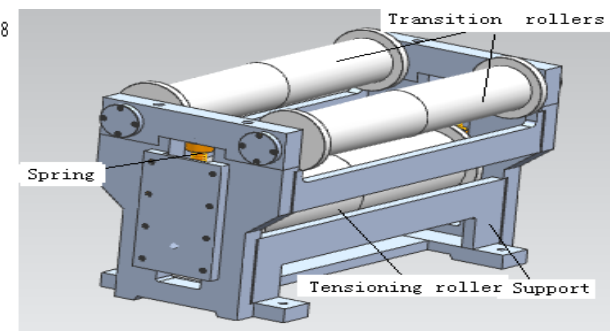

Fig. 3 Automatic tension mechanism

\section{Design of belt tensioning and deviation-rectifying device}

Design of belt tensioning device. At present, friction drive is adopted in the mesh belt sintering furnace. As belt drive, the belt drive system of sintering furnace includes tight edge and loose edge [5]. In belt drive, the elongate of mesh belt which is acted upon by tensile and thermal expansion force acting, will not do harm to the small span transmission. But for large span transmission, the loose edge will be dithering while running. Therefore, the transmission system of sintering furnace is tensioned across the whole span as shown in Fig. 1. On the return section of mesh belt, a series of transition wheels are adopted, and two gravity tensioning devices are placed fore and aft of sintering furnace as shown in Fig. 3. In this way, the transmission belt can always keep tension and the transmission system can run smoothly.

Design of belt deviation-rectifying device. The mesh belt of sintering furnace is designed with big holes and thin silk to make the belt cool down quickly. But the belt with big holes and thin silk is easier to be distorted and deviated from the guide rail in running. The adjustment screws are set on the bearings of the driven roller. It can be adjusted to change the guide angle of roller and make the belt 
run rightly when the belt wander while running, as shown in Fig. 4. The limited guide rails are installed in the both sides of the belt to effectively prevent the belt swing in the furnace and ensure belt conveyor operating well. The transmission system uses spherical bearing which is convenient to install and adjust.

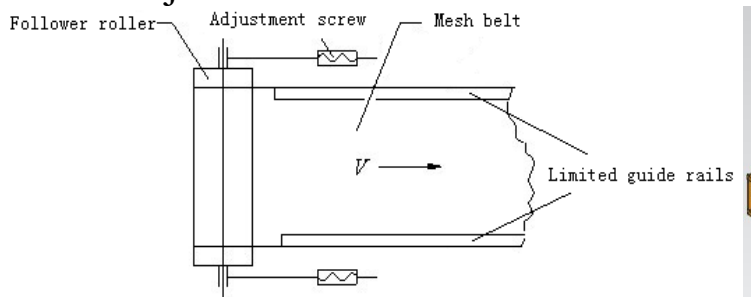

Fig. 4 Schematic of correction device structure

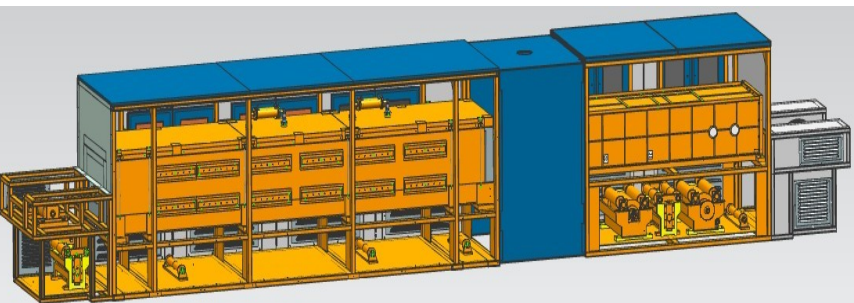

Fig. 5 3-D model of sintering furnace for solar cell

\section{Conclusions}

The power source of the sintering furnace transmission system is designed and calculated. Furthermore, the overall structure of transmission system of sintering furnace is realized and 3-d model of the sintering furnace is established, including the design of deceleration mechanism, tensioning device and deviation-rectifying device (as shown in Fig. 5). The equipment has already been manufactured, and the result indicates that it operates well and achieves the anticipated goal. This method can be extended to other transmission system in continuous high temperature heat treatment equipment.

\section{Acknowledgements}

This work was financially supported by the Specific Project on Energy Saving and Emission Reduction of Shanghai Science and Technology Commission (10dz1204500) and Shanghai Key Subject Construction Project (Y0102).

\section{References}

[1] Xiaoyan Wei, Mingliang Li: J. Coal Technology. Vol. 19(5) (2000), p.54-56.

[2] S.Bhushan, M.Vishal, R.Robert: Using silicon injection phenomenon during fire-through contact formation to improve process control and performance of screen-printed multicrystalline-silicon solar cells. Conference Record of the IEEE Photovoltaic Specialists Conference. (2010), p. 3614-3617.

[3] Techao Chen: J. Equipment for Electronic Products Manufacturing. Vol. 159(2008), p.10-13.

[4] Xiaoguang Hao, Anting Wang, Shiping Zhou, Kai Zhou: J. New Chemical Materials. Vol. 34(9) (2006), p.37-39.

[5] Yong Tang, Zhangqi Yuan, Zuosheng Liao: J. Equipment for Electronic Products Manufacturing. .Vol. 138 (2006), p.52-54. 